\title{
A Replay of Few-Body Reactions in Particle Cosmology
}

\author{
W-Y. P. Hwang ${ }^{a}$, T.-S. H. Lee ${ }^{b}$, and Bo-Qiang $\mathrm{Ma}^{\mathrm{c}}$ \\ anstitute of Astrophysics \& Department of Physics, \\ National Taiwan University, Taipei, Taiwan 106 \\ ${ }^{b}$ Physics Division, Argonne National Laboratory, \\ 9700 South Cass Avenue, Argonne, IL 60439-4843, U.S.A. \\ 'Institute of Theoretical Physics, School of Physics, \\ Peking University, Beijing 100871
}

The existence of the Greisen-Zatsepin-Kuzmin (GZK) cutoff energy for protons does not preclude the possibility of accelerating protons to energies considerably higher than the GZK cutoff. Rather, it indicates that protons being accelerated to above GZK cutoff energies, subsequently propagating in the cosmic medium while interacting with cosmic background photons, become the source of producing other ultra high energy cosmic rays (UHECR's) - some of them, such as GZK neutrinos, could propagate with a much longer mean free path. It is therefore of critical importance to investigate experimentally the neutrino fraction in the high energy cosmic rays, as a function of the cosmic ray energy up to energies beyond the GZK cutoff. In this paper, we wish to demonstrate how the various few-body reactions, so familiar to the audience of the few-body physics community, replay themselves at the highest-energy edge of particle cosmology. As one of our initial attempts, we employ the current state-of-the-art knowledge on photo-pion mucleon reactions to investigate the propagation, in the cosmic medium, of nucleons of energy in the range of $(1-6) \times 10^{20} \mathrm{eV}$, i.e. between the GZK cutoff and $\Lambda \mathrm{K}$ production threshold. A sample numerical result is given for illustration.

\section{Introduction}

Air shower experiments, including Haverah Park [1] and AGASA [2] arrays, have observed, during the last couple of years, about a dozen of cosmic rays with energy in excess of $100 \mathrm{EeV}$, i.e. $10^{20} \mathrm{eV}$. The question regarding what these ultra high energy cosmic rays (UHECR's) really are and how and where they are accelerated to such extraordinarily high energies has already resulted in experimental efforts in building large-scale air shower arrays, such as the 4,000 $\mathrm{km}^{2}$ Pierre Auger Observatory, as well as theoretical attempts to bring together all these puzzles to further our understanding of the Universe [3].

Making use of the celebrated argument, c.f. [3], that, in order to accelerate a proton to energy $E$ in a magnetic field $B$, the size $R$ of the accelerator must be larger than the 
gyroradius of the particle:

$R>R_{\text {gyro }}=\frac{E}{B}$

we obtain the maximum beam energy

$E=\gamma^{-1} B R$,

with the Lorentz $\gamma$-factor accounting for the fact that the cosmic accelerator is often speeding away from us, such as indicated by the Hubble expansion. In the case of an AGN black hole with an approximate black-hole mass of $10^{9} M_{\text {sun }}$, the accelerating region may be more than 10 times away from the event horizon, say $R \approx 10 R_{s}=20 G_{N} M / c^{2}$, with a magnetic field ranging from $\left(10^{3} \sim 10^{12}\right)$ Gauss and $\gamma \approx 1$, the $1 \%$-efficiency acceleration may bring out a proton of energy from $\left(10^{18} \sim 10^{27}\right) \mathrm{eV}$. Such acceleration may take place either in the "jet" region or near the accretion disk (probably sideways if manage to get out). Such argument would suggest that there would be plenty of GZK protons, i.e., those protons of energy greater than the GZK cutoff (as explained immediately below), and as it is believed that there are a huge number of AGN black holes or quasars all over the sky, there would not be preferred directions for UHECR's, except from those nearby AGN's. It would therefore be of some interest to lock in a long-term observation with the known nearby AGN candidates such as M87.

\section{Replay of Few-Body Reactions}

The present universe has a cosmic medium consisting of the $2.73^{\circ} \mathrm{K}$ cosmic microwave background (CMB) photons and the $1.9^{\circ} K$ cosmic background (CB) neutrinos. A cosmic proton with energy greater than $1.10 \times 10^{20} \mathrm{eV}$ will begin "seeing" the CMB photons since the CM energy of $p+\gamma_{C M B}$ exceeds the pion-production $N+\pi$ threshold. The threshold is called the "Greisen-Zatsepin-Kuzmin (GZK)" cutoff [4]. In the cosmic medium, the mean free path for the pion photoproduction reactions,

$$
\begin{aligned}
p+\gamma_{C M B} & \rightarrow n+\pi^{+} \\
& \rightarrow p+\pi^{0},
\end{aligned}
$$

is in the range of a few $M p c[5]$. Of course, neutrons produced in such reactions would decay but, with the Lorentz factor, a GZK neutron has a decay mean free path of about $1 \mathrm{Mpc}$. If GZK neutrons, i.e. of energy greater than the GZK cutoff, are produced, they undergo the following reactions:

$$
\begin{aligned}
n+\gamma_{C M B} & \rightarrow p+\pi^{-} \\
& \rightarrow n+\pi^{0} \\
n & \rightarrow p+e^{-}+\bar{\nu}_{e} .
\end{aligned}
$$

In other words, it transforms back to a proton or a neutron. $\pi^{ \pm}$will decay quickly into $\mu^{ \pm}+\nu_{\mu}$ while $\pi^{0}$ decays into two photons which cannot travel very far in the cosmic medium. The mutual conversion between the proton and neutron will persist until a proton with energy below the GZK cutoff is produced and in the process many other 
particles, especially the neutrinos, appear in the "beam" - neutrinos are stable while electrons and photons eventually get strayed away from the "beam". If the CM energy of the $N+\gamma_{C M B}$ reaches the threshold of the $\Lambda+K$ production, at which the proton is about $7 \times 10^{20} \mathrm{eV}$, more channels open up but the treatment of the problem is similar.

In any event, the size of the acceleration region near an AGN black hole is so much smaller than the GZK mean free paths, that protons may get accelerated to extreme energies but travel with GZK conversion in the cosmic medium. We stress again that the presence of the GZK cutoff does not mean that protons cannot be accelerated to above the GZK cutoff energy. On the other hand, the fact that we have been using possible acceleration near a gigantic black hole as our illustrative example does not exclude other plausible scenarios for producing UHECR's in the literature.

\section{Illustrative Numerical Results}

To further our understanding of the problem, we have employed the state-of-the-art knowledge of the pion photoproduction off nucleons to investigate the propagation in the cosmic medium of a proton of energy in the range of $(1 \sim 6) \times 10^{20} \mathrm{eV}$, i.e. between the GZK cutoff and the $\Lambda K$ production threshold. Presumably, we should also allow the possibility of having protons of energy considerably greater than the $\Lambda K$ threshold, extending to $\left(10^{22} \sim 10^{24} \mathrm{eV}\right)$, but this will make the situation much more complicated, due to the large number of reaction channels involved, and thus we need to leave it as another subject of serious investigation. Indeed, it is also illuminating to have a serious quantitative treatment of the problem in which the energy of the cosmic proton lies in the region where pion photoproduction with CMB photons dominates.

Leaving the explanation of the details of our method to some other article[6], we show in the following an example of our numerical results:

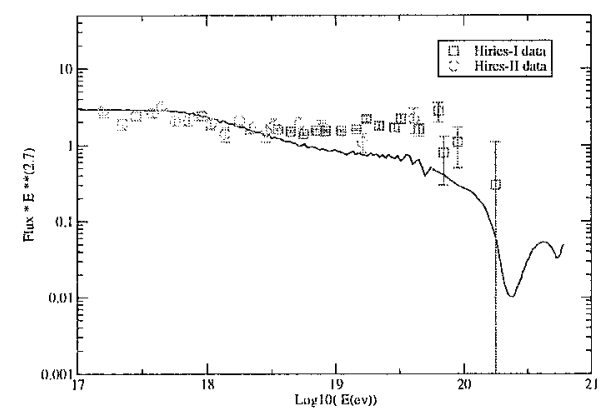

In brief, the figure shown here is obtained by solving numerically the transport equations[7], making use of the cross sections of various pion photo-production reactions mentioned above. Here we make use of the model of Sato and Lee[8], which reproduces the data of $\gamma p \rightarrow \pi^{0} p, \gamma p \rightarrow \pi^{+} n$, and $\gamma n \rightarrow \pi^{-} n$ reactions very well from the threshold up to the $\Delta$ excitation region and can generate the corresponding $\gamma n \rightarrow \pi^{0} n$ cross sections which are not available experimentally, but are needed for our calculations. With 
these input, we can investigate the propagation of cosmic nucleons in the energy region $1.1 \times 10^{20} \mathrm{eV} \leq \mathrm{E} \leq 6 \times 10^{20} \mathrm{eV}$.

The figure shows that we are able to qualitatively reproduce HiRes data with an initial proton spectrum $\Phi_{p}(E, 0) \sim 1 / E^{2.7}$ emitted from a source at a distance $x_{0} \sim 20 \mathrm{Mpc}$ from the earth. The spectrum below GZK cutoff is taken from the calculation of Ref.[9]. The continuation of this low energy spectrum to our results at $\mathrm{E} \geq 1.1 \times 10^{20}$ is taken as a constraint in fitting the data by adjusting the initial proton spectrum and the distance $x_{0}$. For simplicity, we only consider the power-law spectrum $\Phi(E, 0) \sim 1 / E^{n}$ with $n$ varied in fitting the data.

\section{Summary}

In this short article, we wish to demonstrate that the pion photo-production reactions, the celebrated few-body reactions in the few-body physics community, manifest themselves again in the high energy extreme of cosmic rays. It is clear that many more few-body reactions will come into play as the physics at the even more high energies is explored.

\section{Acknowledgement}

We would like to thank K. Nollett for sending us his results of proton spectrum below GZK cutoff. The work of W-Y. P. Hwang is supported in part by the Taiwan CosPA Project of the Ministry of Education of R.O.C. and in part by the grant from the National Science Council of R.O.C. The work by T.-S. H. Lee is supported in part by the U.S. Department of Energy, Nuclear Physics Division (Contract No. W-31-109-ENG), and in part by the Taiwan CosPA Project.

\section{REFERENCES}

1. M. Ave et al., Phys. Rev. Lett. 85, 2244 (2000).

2. See http://www-akeno.icrr.u-tokyo.ac.jp/AGASA/.

3. F. Halzen, "The Highest Energy Cosmic Rays, Gamma Rays, and Neutrinos: Facts, Fancy, and Resolution", arXiv:astro-ph/0111059, 2 Nov 2001; for earlier comprehensive reviews, see, e.g., P. Bhattacharjee and G. Sigl, Phys. Reports 327, 109 (2000) or M. Nagano and A.A. Watson, Rev. Mod. Phys. 72, 689 (2000).

4. K. Greisen, Phys. Rev. Lett. 16, 748 (1966); G.T. Zatsepin and V.A. Kuzmin, Pis'ma Zh. Eksp. Tero. Fiz. 4, 114 (1966) [JETP Lett. 4, 78 (1966)].

5. W-Y. P. Hwang and Bo-Qiang Ma, "Neutrons in the Ultrahigh Energy Cosmic Rays", preprint (2002).

6. W-Y. P. Hwang, H. T.-S. H. Lee, and Bo-Qiang Ma, Manuscript in Preparation.

7. C. T. Hill and D. N. Schramm, Phys. Rev. D31, 564 (1985)

8. T. Sato and T.-S. H. Lee, Phys. Rev. C 54, 2660 (1996)

9. K. Nollett, private communication (2003). 